Vol: 03, Pg:27-29, Yr:2022

\title{
Antibiofilm activity of Phytocompoundsagainst of Staphylococcus aureusBiofilm forming Protein - In silicostudy
}

\author{
Anuradha Ghosal ${ }^{1}$, Rupak Roy ${ }^{1}$, Komal Sharma ${ }^{1}$, Priya Mitra ${ }^{1}$, Kunal Vora ${ }^{1 *}$ \\ ${ }^{1}$ SHRM Biotechnologies Pvt Ltd, Kolkata, West Bengal, India \\ *Corresponding: kunal.vora@shrmbio.com
}

\begin{abstract}
Biofilms are the syntrophic consortia of microbial sessile colonies that remain adhered to the biotic and abiotic surface with the help of self-secreted polymeric substances known as extracellular polymeric substances (EPS). The biofilms developed by the group of Gram-positive bacteria help them to survive within extremes of environmental conditions. This paper, insilico studies were performed on biofilm forming proteins to study the role of various phytocompounds in eradication of biofilm formationby Staphylococcus aureus. It was observed that nimbin showed maximum interaction with the biofilm forming proteins of S. aureus.
\end{abstract}

Keywords: Biofilm, S. aureus, phytrocompounds, molecular interaction, antibiofilm

\section{Introduction}

Biofilms are formed by the adherent group of sessile communities of micro-organisms those remain embedded by a matrix of extracellular polymeric substance(EPS). The adherent cells stick to biotic and abiotic surfaces with the help of extracellular polymeric substances (1). The EPS not only provides nourishment to the developing sessile cells but also prevents the penetration of drugs resulting in the development of muti-drug or antimicrobial resistance (2).Staphylococcus aureus is Gram-positive, potent nosocomial and biofilm forming microbial specie that possesses the ability to adhere on both biotic and abiotic surfaces and result in the development of potent nosocomial infections $(3,4)$. This has resulted in the development of alternative therapeutics for the purpose of preventing the biofilm associated chronic infections (5). This work emphasizes on the use of 
alternative therapeutics comprising of phytocompounds in treating biofilm associated chronic infections by $S$. aureus.

\section{Materials and methods}

\subsection{Docking}

Docking has been done between the bioactive compounds against the biofilm forming protein of $S$. aureus using AutoDock Vina (6). The interaction between the proteins has been checked.

\section{Results and Discussion}

The molecular docking interactions showed that nimbin showed maximum inhibition of the biofilm forming protein of $S$. aureus in comparison to other phytocompounds that interacted with the protein. (Table 1, Figure $1)$.

Table 1: Binding energy of the molecular docking interaction between the biofilm forming protein and phytocompounds

\begin{tabular}{|l|l|l|l|}
\hline Organism & $\begin{array}{l}\text { Biofilm forming } \\
\text { protein }\end{array}$ & Phytocompound & $\begin{array}{l}\text { Binding Energy } \\
\text { (Kcal/mole) }\end{array}$ \\
\hline \multirow{3}{*}{ Staphylococcus aureus } & 3 TIP & Curcumin & -6.00 \\
\cline { 3 - 4 } & & Eugenol & -4.09 \\
\cline { 3 - 4 } & & Quercetin & -5.75 \\
\cline { 3 - 4 } & & Nimbin & -6.26 \\
\hline & & Gingerol & -5.62 \\
\hline
\end{tabular}

B
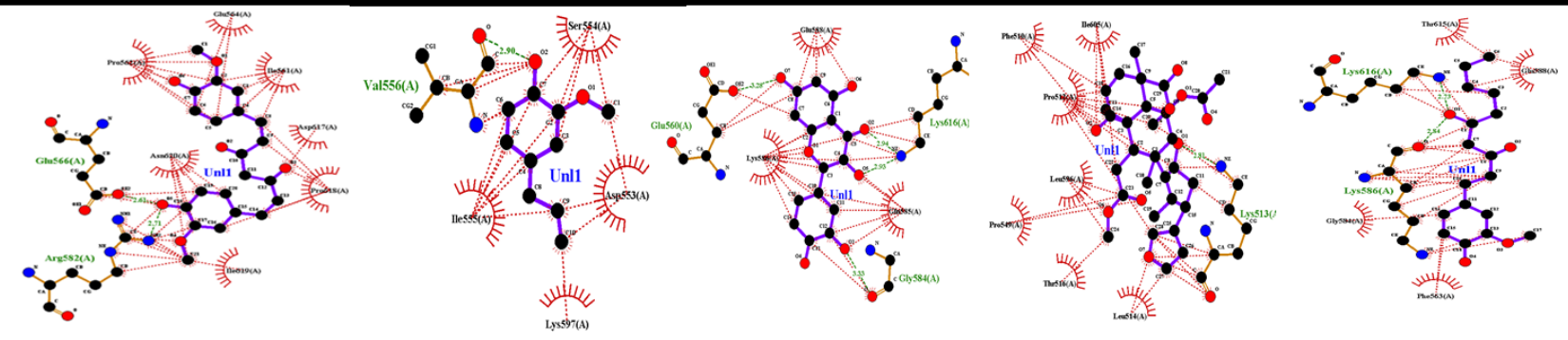

Figure 1: Molecular docking interaction of the biofilm forming proteins of S. aureus with the phytocompounds. 


\section{Conclusion}

The study showed nimbin showed maximum interaction with the biofilm forming proteins of S. aureus. This depicts that phytocompounds can be used as alternate therapeutics in the inhibition of the bifilm formed by major organisms.

\section{References}

1. Lahiri, D., Dash, S., Dutta, R. et al. Elucidating the effect of antibiofilm activity of bioactive compounds extracted from plants. $J$ Biosci 44, 52 . https://doi.org/1 0.1007/12038-019-9868-4, 2019

2. Lahiri, D., Nag, M., Dutta, B. et al. Catechin as the Most Efficient Bioactive Compound from Azadirachta indica with Antibiofilm and Anti-quorum Sensing Activities Against Dental Biofilm: an In Vitro and In Silico Study. Appl Biochem Biotechnol 193, 1617-1630. https://doi.org/10.1007/s 12010-02103511-1, 2021

3. Ray R.R., Lahiri D., Chatterjee A., Banerjee P. Bacteria and Biofilms as Natural Inhabitants of Our Body. In: Ray R.R., Nag M., Lahiri D. (eds) Biofilm-Mediated Diseases: Causes and Controls. Springer, Singapore. https://doiorg/10.1007/978-981-160745-5_3, 2021

4. Ray R.R., Lahiri D., Chatterjee A., Banerjee P. Bacteria and Biofilms as Natural Inhabitants of Our Body. In: Ray R.R., Nag M., Lahiri D. (eds) Biofilm-Mediated Diseases: Causes and Controls. Springer, Singapore. https://doi. org/10.1007/978-981-160745-5_3. 2021.

5. Ratha B.N., Lahiri D., Ray R.R. Inhibition of Biofilm Formation. In: Ray R.R., Nag M., Lahiri D. (eds) Biofilm-Mediated Diseases: Causes and Controls. Springer, Singapore. https://doi.org/10.1007/978-981160745-5_9. 2021.

6. Jørgensen, Nis; Zobek, Natalia; Dreier, Cindy; Haaber, Jakob; Ingmer, Hanne; Larsen, Ole; Meyer, Rikke. Streptokinase Treatment Reverses Biofilm-Associated Antibiotic Resistance in Staphylococcus aureus. Microorganisms, 4(3), 36.2016 